\title{
Helical type coronary bypass graft performance: Experimental investigations
}

\author{
Sandor I. Bernad ${ }^{\mathrm{a}}$, Alin I. Bosioc ${ }^{\mathrm{b}}$, Elena S. Bernad ${ }^{\mathrm{c}, *}$ and Marius L. Craina ${ }^{\mathrm{c}}$ \\ ${ }^{a}$ Romanian Academy-Timisoara Branch, Centre for Fundamental and Advanced Technical Research, \\ Bd. Mihai Viteazul 24, RO-300223, Timisoara, Romania \\ ${ }^{b}$ University Politehnica Timisoara, Department of Hydraulic Machinery, Bd. Mihai Viteazu 1, RO- \\ 300222, Timisoara, Romania \\ ${ }^{c}$ University of Medicine and Pharmacy "Victor Babes" Timisoara, Universitary Clinic "Bega", P-ta \\ Eftimie Murgu 2, RO-300041, Timisoara, Romania
}

\begin{abstract}
Optimal graft design has been an objective of many researchers to find correlations between hemodynamics and graft failure. Compared to planar grafts, the helical graft configurations improve hemodynamic performance including the promotion of flow mixing and reduction of flow stagnation regions. In order to evaluate the advantages and disadvantages of the suggested helical type bypass graft model in comparison to a conventional bypass graft configuration, three experimental models were designed and evaluated. The character of complex vortex structures created in the area between the heel and the occluded section depends on the flow parameters (in the case of the straight graft). We have identified two vortices in the symmetrical plane (proximal and distal to the anastomosis). In the new design of the two-turn helical graft, the stagnation point is eliminated from the anastomoses at different time intervals compared to the conventional straight bypass model The present study indicated that the magnitude of the pressure drop along a helical graft was considerably increased compared to a traditional graft which, while still physiologically advantageous, can be surpassed by an optimal geometry model.
\end{abstract}

Keywords: Bypass graft, helical graft, hemodynamics, pressure drop, anastomosis

\section{Introduction}

It is generally accepted that hemodynamic analysis is imperative to the design of coronary bypass grafts [1]. In recent years, graft design has been a popular research objective to discover potential correlations between hemodynamics and graft failure [2,3]. Researchers have attempted various improvements to increase the hemodynamic performance of the implanted grafts. Numerical studies and in vitro investigations have confirmed that graft flow patterns are strongly dependent on local geometry [4].

\footnotetext{
* Address for correspondence: Elena S. Bernad, University of Medicine and Pharmacy "Victor Babes" Timisoara, Universitary Clinic "Bega", P-ta Eftimie Murgu 2, RO-300041, Timisoara, Romania. Tel.: +40-256-490810; Fax: +40-256490810; E-mail: ebernad@yahoo.com.
}

0959-2989/15/\$35.00 @ 2015 - IOS Press and the authors. 
Compared to traditional planar grafts, non-planar graft configurations improve hemodynamic environments including the promotion of flow mixing and the reduction of flow stagnation regions, but also increases the likelihood of injury to host artery vessels [5-8]. Caro et al. [2] conducted animal research on small-amplitude helical technology (SMAHT) conduits and numerically simulated steady flow in a four-turn helical graft. Morbiducci et al. [9] investigated the existence of a relationship between helical structure and atherogenesis of the vascular wall in aorto-coronary bypass models. Zheng et al. [10] numerically simulated both steady and unsteady flows in helical grafts [11]. Preliminary studies [12-16] suggested that the helical type flow appeared promising in the prevention of intimal hyperplasia (IH) and thrombosis but demonstrated an increased pressure drop compared to a conventional graft [14].

The primary goal of this study was to increase understanding of the flow mechanisms of helical grafts in order to improve the design of bypass grafts. Traditional flow parameters such as stagnation point and recirculation length were calculated for the comparison. Pressure drops along both straight and helical graft types were investigated in detail.

\section{Methods}

Encouraged by the findings of previous research, $[3,8,17,18]$ steady and unsteady flows in helical type bypass grafts were experimentally investigated in both straight and two-turns helical grafts.

In order to compare the advantages and disadvantages of the helical bypass graft model and a conventional bypass graft configuration, three experimental models were designed and evaluated:

(a) a conventional straight graft configuration (Figure 1)

(b) a two-turn helical-type graft (Figure 1)

(c) a four-turn helical-type graft

The geometric shape of the aorto-coronary bypass grafts are illutrated in Figure 1; geometric dimensions of the aorto-coronary bypass models are illustrated in Table 1 . The artery model is assumed to be a tapered straight vessel with proximal total occlusion. The graft angle $(\theta)$ is selected as a parameter and set at $60^{\circ}$. The characteristic parameters of a two-turn helical tube are as follows: internal diameter $\mathrm{D}=8 \mathrm{~mm}$, pitch of coil $\mathrm{p}=20 \mathrm{~mm}(2,5 \mathrm{xD})$, coil length $=40 \mathrm{~mm}$ (Figure 1), coil diameter $=9 \mathrm{~mm}$.

\subsection{Experimental setup}

A schematic of the experimental system is presented in Figure 2. A glycerine-water mixture of $60 \%$ glycerine and $40 \%$ water by weight was selected as the working fluid.

The flow experiment was performed under physiological conditions [8]. The mean flow velocity is $0.144 \mathrm{~m} / \mathrm{s}$. The mean Reynolds number $\mathrm{Re}=260$ (based on graft diameter and blood viscosity). The maximum Reynolds number during the cycle was $R e=612$. Assuming a heart rate of 60 beats per minute, the period of each cycle is 1 second.

The experimental graft models were manufactured from glass tubing with an internal diameter $\mathrm{D}=8$ $\mathrm{mm}$, fashioned into straight and helical configurations with a straight segment approximately $80 \mathrm{~mm}$ proximally and distally (Figure 1).

The flow system consisted of a constant storage head tank, test section, floating ball flowmeter, collecting tank, and variable-speed centrifugal pump. A computer-controlled pump was used to generate the inlet flow waveform. The amplitude of flow fluctuation introduced by the pump was a function of 


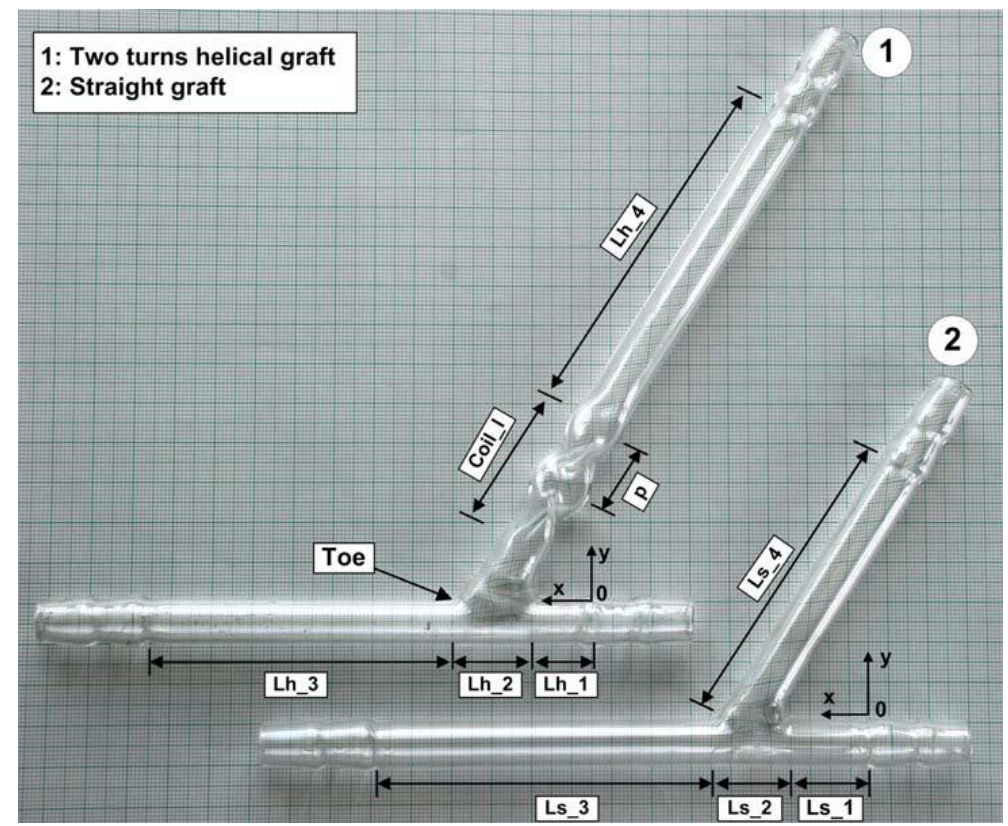

Fig. 1. Experimental bypass models manufactured from glass tubing with constant internal diameter of $8 \mathrm{~mm}$. (a) Two-turn helical graft model; (b) Straight graft model.

Table 1

Bypass parameters (mm)

\begin{tabular}{|c|c|c|c|c|}
\hline Parameters & Ls_1/Lh_1 $[\mathrm{mm}]$ & Ls_2/Lh_2 $[\mathrm{mm}]$ & Ls_3/Lh_3 $[\mathrm{mm}]$ & Ls_4/Lh_4 [mm] \\
\hline Straight bypass & 15 & 20 & 80 & 80 \\
\hline Two-turn helical bypass & 15 & 20 & 80 & 80 \\
\hline
\end{tabular}

the difference between the upstream and downstream flow resistances. The amplitude of flow fluctuation could be predetermined by adjusting the valves upstream and downstream of the test section, thus adjusting the resistance (Figure 1). While collecting experimental measurements, the waveform at flowmeter F2 was identical to the generated waveform at flowmeter F1 with no phase shift.

The mean flow rate (upstream of the test section) was measured by a metric size 10 rotameter with a stainless steel float (flowmeter F1, illustrated in in Figure 1). The rotameter leg additionally prevented air from travelling upstream from the flow outlet. A blood analog fluid was prepared with dynamic viscosity $(\mu)$ of 0.00408 Pa.s and a density $(\rho)$ of $1050 \mathrm{~kg} / \mathrm{m} 3$. The flow visualization study was performed using the injection of a bolus of ink into flowing fluid at various Reynolds numbers.

\section{Results}

Investigated bypass graft configurations are proposed to benefit the flow field in various ways including the maintenance of a uniform, smooth flow in the anastomotic region, causing lesser impact on the artery bed and less or no vortex at the heel region of the anastomosis [18].

Coronary artery bypass grafts (CABG), are involved in several types of anatomical complications including thrombosis and stenosis. Previous literature determined that after vascular surgery, the flow 


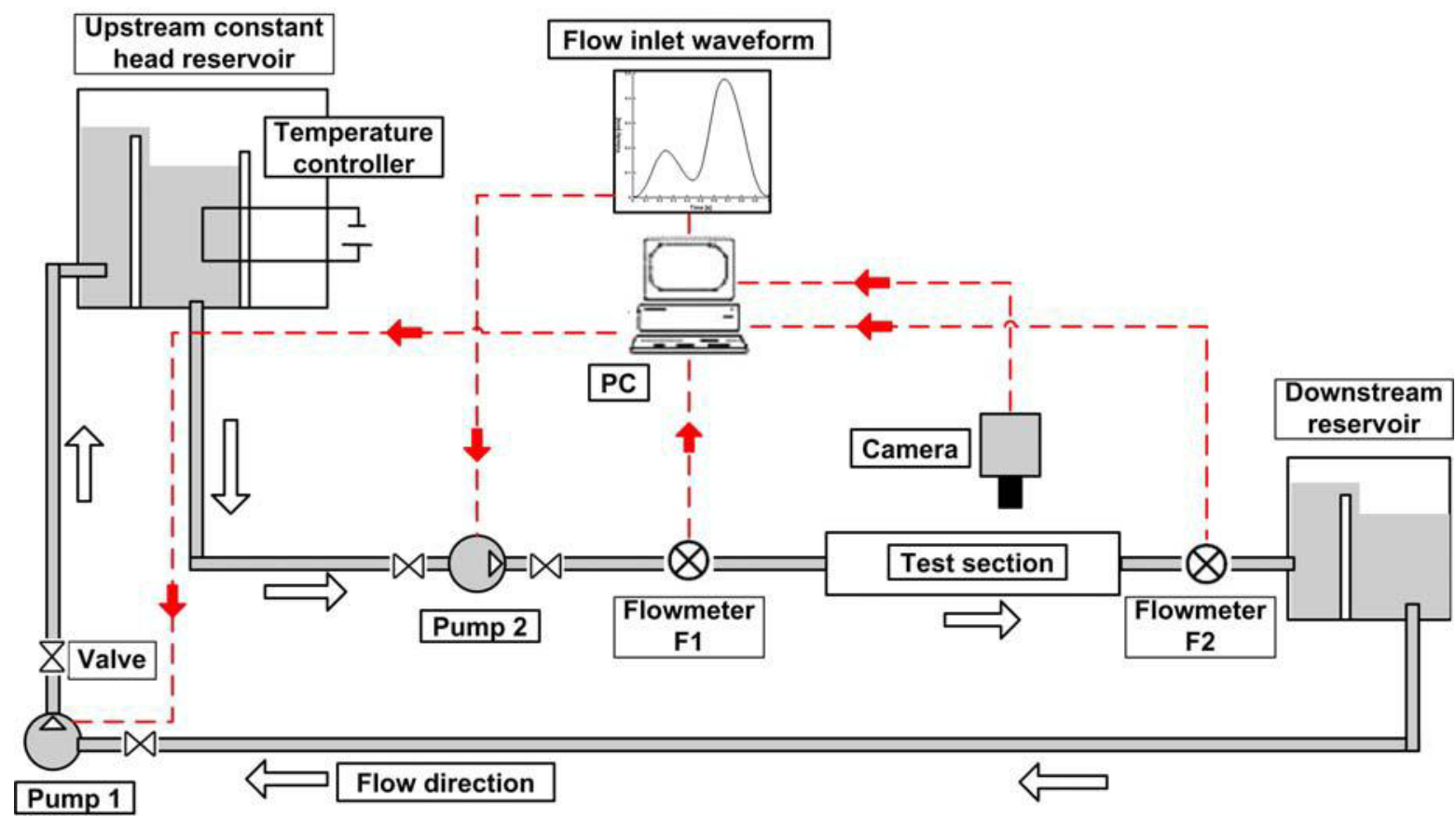

Fig. 2. Experimental setup for investigation of straight and helical type grafts. Steady and pulsatile waveforms used as velocity input signal.

field may play a modulating role. In a case where intimal hyperplasia (IH) is mainly located in regions of low wall shear stress and increased residence time [6, 15, 19], helical flow tends to reduce unfavorable hemodynamic conditions $[9,20,21]$.

In order to focus on the effects of the bypass geometry, all experiments were carried out under the same flow conditions and graft angle. Steady flow in the straight and two-turn helical models is illustrated in Figure 3 with a Reynolds number $R e=298$. Both geometries demonstrate the presence of the primary vortex in the proximal part of the host artery. The straight model was particularly susceptible to particle deposition at the toe of the graft, and may therefore be more susceptible to thrombosis. The vortex zone (V3) for the straight graft and the vortex zone V1 for the two-turn helical graft is shown in Figures 3(a) and 3(b), respectively.

Note the presence of the vortex (V2) in the distal artery as shown in Figure 3(a) and the strong helical flow in the bypass graft as shown in Figure 3(b). The primary vortex (V1) in both cases is located near the anastomosis and rotates counterclockwise as viewed in the photograph, whereas the more distal secondary vortex rotates clockwise as shown by vortex V2 in Figure 3(a).

The character of complex vortex structures created in the area between the heel and occluded section is dependent on the flow parameters. We can identify two vortices in the symmetrical plane, as illustrated by vortices V1 and V3 in Figure 3(a). The shape of these vortices is strongly dependent on the occlusion distance from the anastomosis.

The flow outside the plane of symmetry moves in a helical vortex structure with a variable spiral angle, as shown in Figure 3(a), which create secondary flow in the cross section [8]. The secondary flow intensity decreases downstream and the velocity profile gradually forms a developed laminar velocity profile [17]. 

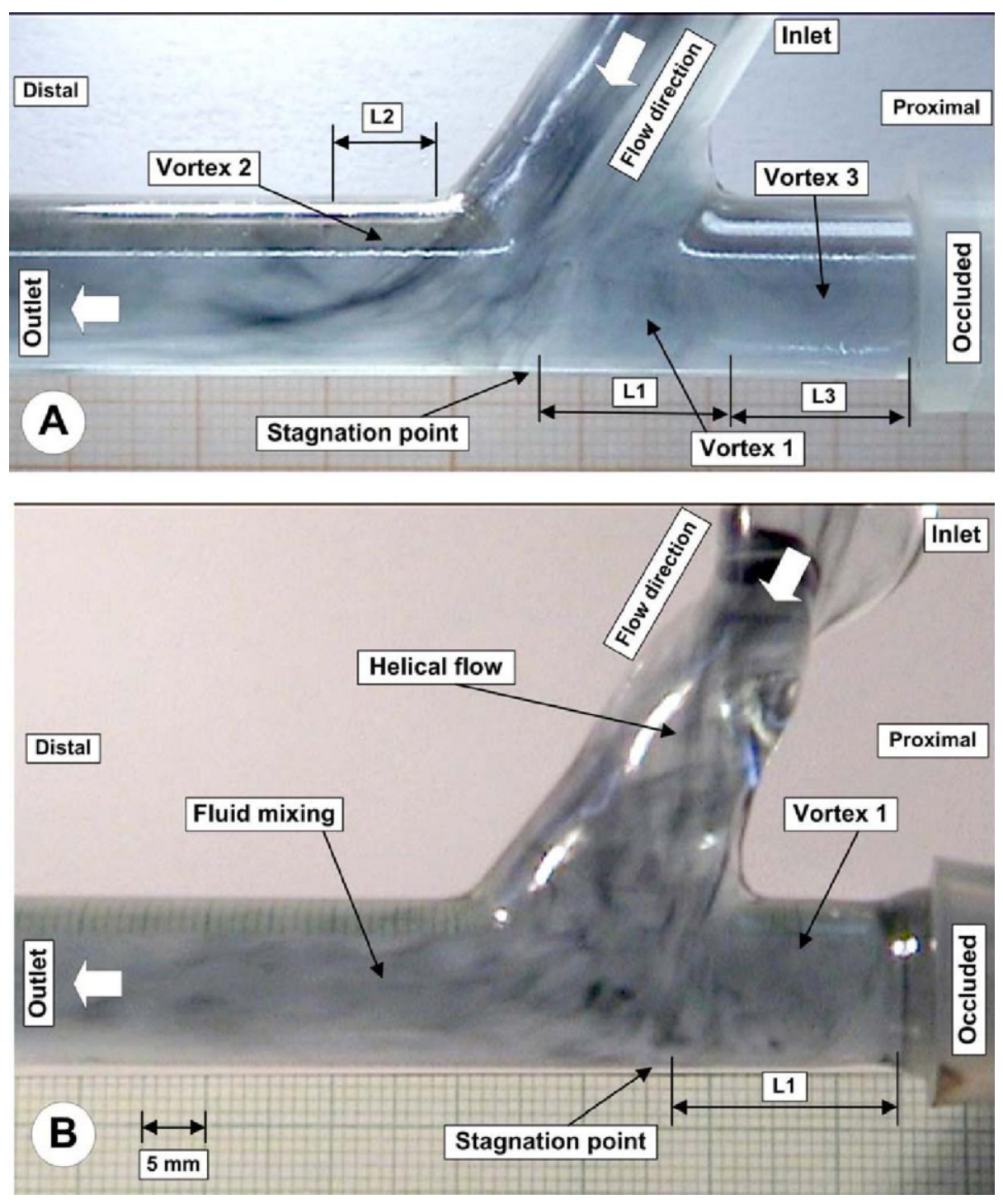

Fig. 3. (a) Flow pattern in straight bypass graft demonstrating secondary flow with spontaneous, periodic disturbances in the distal part of the host artery; (b) Flow pattern in two-turn helical bypass graft, generating only one vortex.

There is also an area with a strong velocity gradient between the main flow and the vortex structure, as shown in Figure 3(a). The size and position of this area is time-dependent for unsteady flow conditions. In this area the magnitude of WSS (wall shear stress) is maximal due to the large velocity gradient. Maximum WSS is time-dependent and shifts along the x-axis (Figure 1) both downstream and upstream, causing wall shear stress oscillation.

In Figure 3(b) the absence of a recirculation region may be advantageous due to reduced contact between the blood and the artificial graft surface, therefore minimizing the risk of thrombosis.

When arterial flow reaches the anastomosis, it enhances the stream from the graft and helps to direct the graft flow into the coronary artery, as shown in Figure 3(b). The point of flow separation for both investigated configurations is more clearly shown in Figure 3 (position of the stagnation point). 

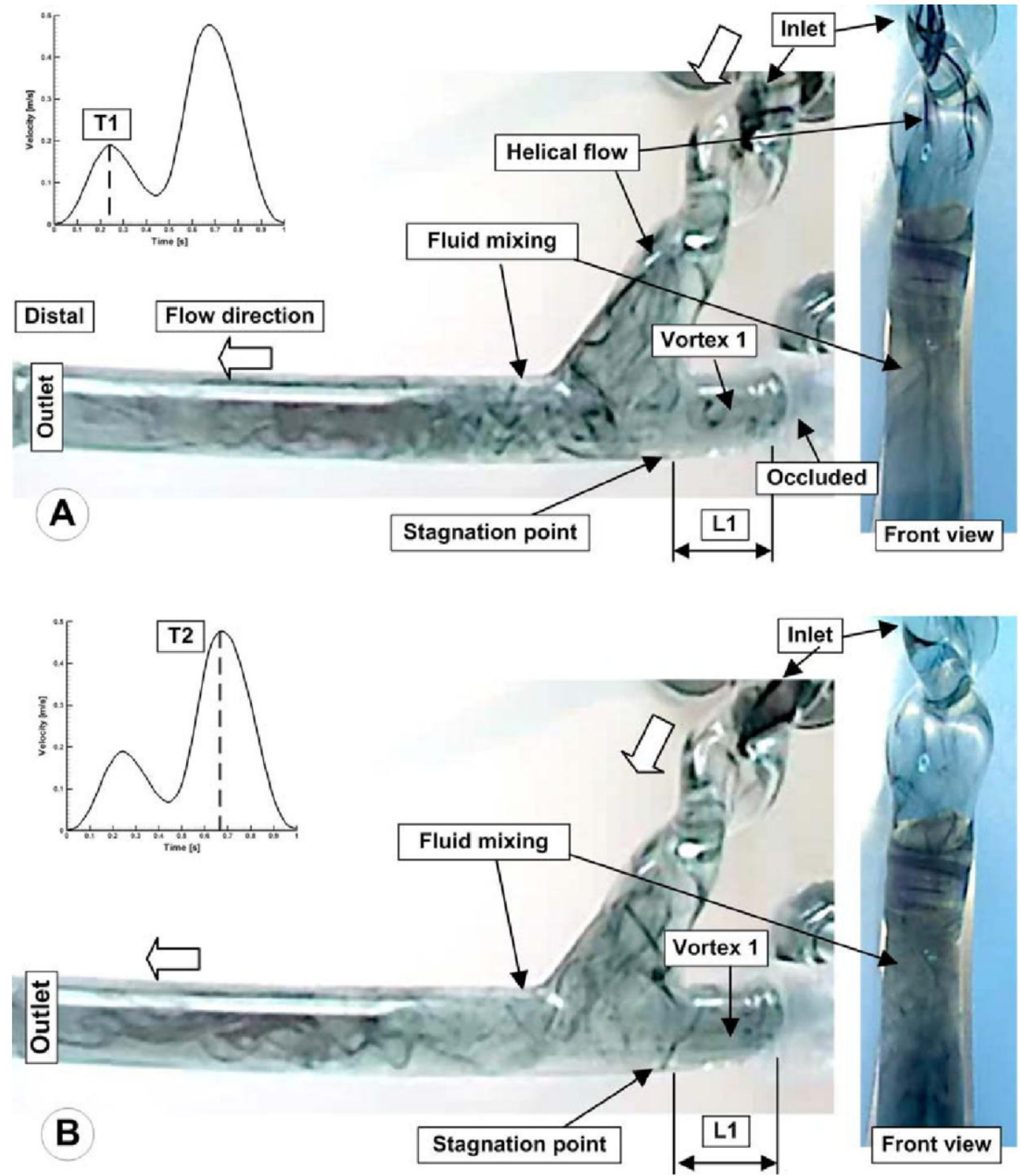

Fig. 4. Comparison between flow pattern in the helical type bypass graft corresponding to the different time steps. 60-degree anastomosis with the two-turn helical graft, under pulsatile flow conditions corresponding to the (a) first and (b) second peaks of the velocity waveform.

Morbiducci et al. [9] demonstrated evidence of a change in flow character once it has impacted the junction floor; thus the impact of flow on the junction floor may be a contributing factor to graft failure. Hence, having a smoothened flow and a lesser impact on the floor of the coronary artery is a demonstrated advantage of the new CABG design.

Figures 4(a) and 4(b) illustrate the flow structure in the $60^{\circ}$ anastomosis with the two-turn helical graft, under pulsatile flow conditions corresponding to the first and second peaks of the velocity 
waveform. During the experimental process, input velocity waveform was generated, corresponding to the diastolic dominant flow demonstrated in Figure 4, in which time T1 and T2 correspond to the systolic peak and diastolic peak, respectively. Note the presence of a single vortex in the occluded part of the bypass graft, with strong helical flow in the graft artery observed during both time measurements; the secondary vortex (V1) rotated counterclockwise, as shown in Figures 4(a) and 4(b). In the anastomosis domain, a recirculation was observed near the occluded end of the artery. This recirculation forces the flow into the perfused distal host coronary artery as indicated in Figures 4(a) and 4(b).

In the new design of the two-turn helical graft, the stagnation point is eliminated from the anastomoses at different time intervals as compared to the conventional straight bypass model. The stagnation point is always associated with the low wall shear stress (WSS) and high spatial wall shear stress gradient (WSSG) regions, contributing to IH and the development of atherosclerosis. Hence, the absence of a stagnation point is another positive feature of the helical graft design.

Using the four-turn helical graft significantly increased the fluid mixing in both the graft and the native artery. Additionally, flow hemodynamic characteristics were influenced by the geometry parameter of the helical part (pitch, coil diameter, and number of coils).

In the new design, there is no vortex formation at the toe of the anastomosis within the coronary artery, resulting in a relatively higher WSS along the artery walls and bed. The presence of a vortex increases the time of local particle residence, which is associated with sites of IH development and may result in platelet activation and fibrin thrombus formation. The absence of vortex formation is another positive feature of the helical graft model.

\section{Discussion}

The goal of this paper is to investigate whether the helical type bypass graft is hemodynamically beneficial to coronary bypass patency, compared to the traditional straight graft type.

On the whole, the geometry of the helical CABG is out of the plane of symmetry, in contrast to the in-plane geometry of the traditional CABG. The three-dimensional and the swirling flow represent basic flow regimes in the helical type CABG. The swirling flow induced in-plane mixing, decreasing the platelet concentration near the floor and suppressing the interaction of platelets with the bypass wall.

Previous literature demonstrated that the helical type flow protects the graft wall from damage by reducing lateral forces. Additionally, swirling flow provides a smooth and even wash of the blood inside the artery, and prevents the transition from laminar flow to turbulence that helps maintain flow stability and eliminate regions of flow stagnation [15, 22].

The flow analysis revealed a strong secondary flow in the helical graft which contributed to the flow mixing between the low-momentum fluid nearer to the surface and the high-momentum fluid at the center.

A possible disadvantage of the proposed helical type graft includes the predication of an increased pressure drop, revealing increased flow resistance at a fixed flow rate that results in increased blood pressure, which may facilitate the development and progression of IH and thrombosis [23].

In order analyze the effects of helical bypass on the pressure drop, three bypass graft models were chosen for calculation of area-averaged pressures at two moments: a) first (systolic) peak, as shown in Figure 4(a); b) maximum velocity of the cardiac cycle, measured by the second (diastolic) peak as shown in Figure 4(b). The pressure drop varied with time and peaked at maximum velocity. Generally, 


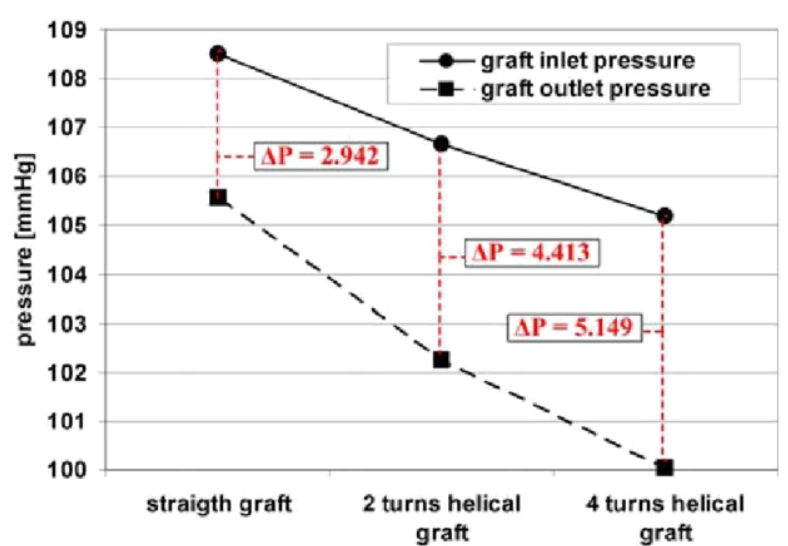

Fig. 5. Comparison of the pressure drop for investigated bypass geometries at the first peak of the cardiac cycle (peak systole).

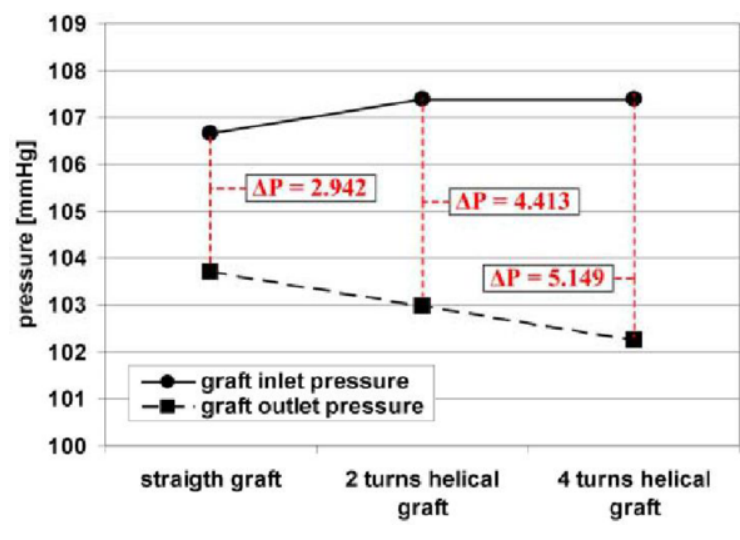

Fig. 6. Comparison of the pressure drop for investigated by-pass geometries at the maximum velocity - second peak of the cardiac cycle (corresponding to Figure 4(b)).

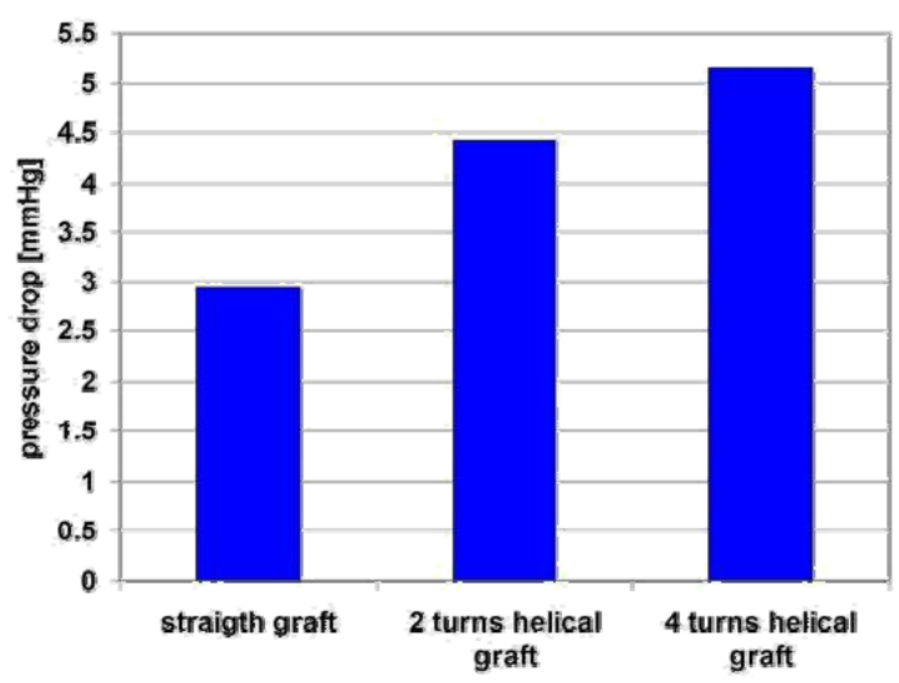

Fig. 7. Pressure drop variations for investigated bypass graft models.

the helical bypass did increase the pressure drop compared to the traditional straight CABG, the percentage increase varying with the cycle. A maximum percentage increase of $75 \%$ occurred at the moment of maximum velocity. The pressure drop along the four-turn helical bypass graft was 5.149 $\mathrm{mm} \mathrm{Hg}$, and the two-turn helical graft demonstrated a pressure drop increase of $50 \%$ compared to the straight graft (Figures 5, 6 and 7).

\section{Conclusions}

Introducing helical turns in the design of the bypass influences the hemodynamics in the graft and the draining artery. Helical flow generation generates a complex flow field which reduces the area of unfavorable wall shear stress conditions associated with the risk of intimal hyperplasia. 
For the helical design, some advantages were observed in the flow field. Experimental investigation results demonstrate that the new model provides:

(i) a uniform and smooth flow at the anastomosis, with no vortex in the toe region of the bypass graft;

(ii) a spare route for the blood flow to the coronary artery, to avoid re-operation in case of restenosis in either of the anastomoses;

(iii) good hemodynamic parameters inside to the coronary artery bed and in the heel region of the anastomosis.

Our results presented in this paper indicated that for helical type grafts, the magnitude of the pressure drop along a helical graft was increased compared to a conventional graft. According to our results the helical type CABG increased the pressure drop by a maximum of $75 \%$ compared to the traditional graft, which is physiologically impractical, but may be improved by optimizing the geometric model. We expect that a helical-type bypass graft may decrease platelet adhesion and clot formation on the graft surface and reduce the risk of IH at the distal anastomosis region.

Quantitative analysis presented in this paper increases understanding of the impact and potential advantages of the helical-type graft to improve the traditional bypass graft design.

\section{References}

[1] P.M. O'Flynn, G. O'Sullivan and A.S. Pandit, Methods for three dimensional geometric characterization of the arterial vasculature, Annals of Biomedical Engineering 35 (2007), 1368-1381.

[2] C.G. Caro, J.C. Nick and W. Nick, Preliminary comparative study of small amplitude helical and conventional ePTFE arteriovenous shunts in pigs, Journal of the Royal Society Interface 2 (2005), 261-266.

[3] A.F. Totorean, A.I. Bosioc, S.I. Bernad and R. Susan-Resiga, Identification and visualization of vortices in by-pass graft flow, Proceedings of the Romanian Academy Series A 15 (2014), 52-59.

[4] J.S. Cole, J.K. Watterson and O. Reilly, Numerical investigation of the haemodynamics at a patched arterial bypass anastomosis, Medical Engineering \& Physics 24 (2002), 393-401.

[5] S. Giordana, S.J. Sherwin, J. Peiro, D.J. Doorly, J.S. Crane, K.E. Lee, N.J.W. Cheshire and C.G. Caro, Local and global geometric influence on steady flow in distal anastomoses of peripheral bypass grafts, Journal of Biomechanical Engineering 127 (2007), 1087- 1098.

[6] F. Loth, P.F. Fischer and H.S. Bassiouny, Blood flow in end-to-side anastomoses, Annual Review of Fluid Mechanics 40 (2008), 367-393.

[7] K.V. Canneyt, U. Morbiducci, S. Eloot, G. de Sanstis and P. Verdock, A computational exploration of helical arteriovenous graft designs, Journal of Biomechanics 46 (2013), 345-353.

[8] S.I. Bernad, A. Bosioc, E.S. Bernad and M.L. Craina, Comparison between experimentally measured flow patterns for straigth and helical graft, Bio-Medical Materials and Engineering 24 (2014), 853-860.

[9] U. Morbiducci, R. Ponzini, M. Grigionid and A. Redael, Helical flow as fluid dynamic signature for atherogenesis risk in aortocoronary bypass. A numeric study, Journal of Biomechanics 40 (2007), 519-534.

[10] T.H. Zheng, Y.B. Fan, Y. Xiong, W.T. Jiang and X.Y. Deng, Hemodynamic performance study on small diameter helical grafts, ASAIO J 55 (2009), 192-199.

[11] T. Zheng, W. Wang, W. Jiang, X. Deng and Y. Fan, Assessing hemodynamic performances of small diameter helical grafts: transient simulation, Journal of Mechanics in Medicine and Biology 12 (2012), 1250008.

[12] G. Coppola and C. Caro, Oxygen mass transfer in a model threedimensional artery, Journal of the Royal Society Interface 5 (2008), 1067-1075.

[13] A.N. Cookson, D.J. Doorly and S.J. Sherwin, Mixing through stirring of steady flow in small amplitude helical tubes, Annals of Biomedical Engineering 37 (2009), 710-721.

[14] Z. Chen, Y.B. Fan, X.Y. Deng and Z. Xu, Swirling flow can suppress flow disturbances in endovascular stents: A numerical study, ASAIO J 55 (2009), 543-549.

[15] F. Zhan, Y.B. Fan and X.Y. Deng, Swirling flow created in a glass tube suppressed platelet adhesion to the surface of the tube: Its implication in the design of small-caliber arterial grafts, Thrombosis Research 125 (2010), 413-418. 
[16] A. Sun, Y. Fan and X. Deng, Numerical comparative study on the hemodynamic performance of a new helical graft with noncircular cross section and swirlgraft, Artificial Organs 34 (2010), 22-27.

[17] E.S. Bernad, S.I. Bernad and M.L. Craina, Hemodynamic parameters measurements to assess severity of serial lesions in patient specific right coronary artery, Bio-Medical Materials and Engineering 24 (2014), 323-334.

[18] S.I. Bernad, A. Bosioc, A. Totorean, R. Stanciu and E.S. Bernad, Vortices in by-pass graft flow, AIP Conference Proceedings of 11th International Conference of Numerical Analysis and Applied Mathematics (ICNAAM) 1558 (2013), 160-163.

[19] S.W. Lee, D. Smith, F. Loth, P. Fischer and H. Bassiouny, Importance of flow division on transition to turbulence within an arteriovenous graft, Journal of Biomechanics 40 (2007), 981-992.

[20] U. Morbiducci, R. Ponzini, G. Rizzo, M. Cadioli, A. Esposito, F. Montevecchi and A Redaelli, Mechanistic insight into the physiological relevance of helical blood flow in the human aorta: an in vivo study, Biomechanics and Modeling in Mechanobiology 10 (2011), 339-355.

[21] I.C. Campbell, L.H. Timmins, D.P. Giddens, R. Virmani, A. Veneziani, S.T. Rab, H. Samady, M.C. McDaniel, A.L. Finn, W.R. Taylor and J.N. Oshinski, Computational fluid dynamics simulations of hemodynamics in plaque erosion, Cardiovascular Engineering and Technology 4 (2013), 464-473.

[22] N. Watanabe, T. Masuda, T. Iida, H. Kataoka, T. Fujimoto and S. Takatani, Quantification of the secondary flow in a radial coupled centrifugal blood pump based on particle tracking velocimetry, Artificial Organs 29 (2005), 26-35.

[23] A.K. Politis, G.P. Stavropoulos, M.N. Christolis, F.G. Panagopoulos, N.S. Vlachos and N.C. Markatos, Numerical modeling of simulated blood flow in idealized composite arterial coronary grafts: Steady state simulations, Journal of Biomechanics 40 (2007), 1125-1136. 